CQUeST-2009-0306

\title{
On Charged Lifshitz Black Holes
}

\author{
Da-Wei Pang ${ }^{\dagger}$ \\ † Center for Quantum Spacetime, Sogang University \\ Seoul 121-742, Korea \\ pangdw@sogang.ac.kr
}

\begin{abstract}
We obtain exact solutions of charged asymptotically Lifshitz black holes in arbitrary $(d+2)$ dimensions, generalizing the four dimensional solution investigated in 0908.2611[hep-th]. We find that both the conventional Hamiltonian approach and the recently proposed method for defining mass in non-relativistic backgrounds do not work for this specific example. Thus the mass of the black hole can only be determined by the first law of thermodynamics. We also obtain perturbative solutions in five-dimensional Gauss-Bonnet gravity. The ratio of shear viscosity over entropy density and the DC conductivity are calculated in the presence of Gauss-Bonnet corrections.
\end{abstract}




\section{Contents}

1 Introduction

2 The solution and thermodynamics 3

3 Gauss-Bonnet corrections

4 Calculating $\eta / s+10$

5 Conductivity $\quad 14$

6 Summary and discussion 18

\begin{tabular}{ll|} 
References & 20 \\
\hline
\end{tabular}

\section{Introduction}

The AdS/CFT correspondence [1, 2, 3, 4] has been extensively studied and fruitful progress has been made in recent years. In particular, as a strong-weak duality, it has provided a useful tool for studying strongly coupled field theories. Moreover, inspired by condensed matter physics, studies of non-relativistic AdS/CFT correspondence have been accelerated since last year, which may open a new window for investigating physical systems in the real world. For reviews see [5].

In many condensed matter systems near a critical point, there exist field theories with anisotropic scaling symmetry, that is, temporal and spatial coordinates scale differently,

$$
t \rightarrow \lambda^{z} t, \quad x^{i} \rightarrow \lambda x^{i}
$$

where $z$ is called the 'dynamical exponent'. Until now, there are two main concrete examples of the gravity duals of non-relativistic field theories. One is called the Schrödinger case, which was proposed in [6, 7] and the finite temperature generalizations were investigated in [8, 9, 10]. The other is the Lifshitz case, which was obtained in [11]. For general 
$(d+2)$-dimensional spacetime, the dual geometry of Lifshitz fixed points is given by

$$
d s^{2}=L^{2}\left(-r^{2 z} d t^{2}+\frac{d r^{2}}{r^{2}}+r^{2} d \vec{x}^{2}\right)
$$

where $d \vec{x}^{2}=d x_{1}^{2}+\cdots+d x_{d}^{2}$. The scale transformation acts as

$$
t \rightarrow \lambda^{z} t, \quad x \rightarrow \lambda x, \quad r \rightarrow \frac{r}{\lambda}
$$

When $z=1$, it reduces to the usual AdS metric.

When $d=2$, the above geometry can be obtained from a $(3+1)$-dimensional gravity coupled with a negative cosmological constant to abelian two- and three-form field strengths,

$$
S=\int d^{4} x \sqrt{-g}(R-2 \Lambda)-\frac{1}{2} \int * F_{(2)} \wedge F_{(2)}-\frac{1}{2} \int * H_{(3)} \wedge H_{(3)}-c \int B_{(2)} \wedge F_{(2)},
$$

where $F_{(2)}=d A_{(1)}, H_{(3)}=d B_{(2)}$. To obtain such a solution the cosmological constant and the gauge field strengths should take the following values,

$$
\begin{aligned}
& \Lambda=-\frac{z^{2}+z+4}{2 L^{2}}, \quad c=\frac{\sqrt{2 z}}{L} \\
& F_{(2)}=\sqrt{2 z(z-1)} L r^{z-1} d r \wedge d t, \quad H_{(3)}=2 \sqrt{z-1} L^{2} r d r \wedge d x_{1} \wedge d x_{2} .
\end{aligned}
$$

However, it is quite difficult to find analytic black hole solutions which are asymptotically Lifshitz-like. Black hole solutions with $z=2$ in the above $(3+1)$-dimensional theory were investigated in [12] via numerical methods. Lifshitz topological holes were studied in [13] where exact solutions were found in certain specific examples. Black holes in asymptotically Lifshitz spacetimes with arbitrary critical exponent and the corresponding thermodynamic behavior were studied in [14, 15]. Another model of non-relativistic holography was proposed in [16] where exact Lifshitz black hole solutions were obtained. For other recent work on Lifshitz black holes see [17]. It should be pointed out that it is very difficult to embed the Lifshitz background into string theory, although certain string theory duals of Lifshitz-like fixed points were obtained in [18]. Recently some no-go theorems for string duals of non-relativistic Lifshitz-like theories were proposed in [19].

Another type of exact Lifshitz black hole solutions was obtained in [20], where the main purpose was to study holographic superconductors with Lifshitz scaling. In order to realize phase transitions, they added a second Maxwell field $\mathcal{F}_{(2)}$ and a scalar field $\psi$ which was charged under the new gauge field but neutral under the original gauge fields 
$F_{(2)}$ and $H_{(3)}$. It was observed that these two additional fields were sufficient to ensure a superconducting phase transition characterized by Lifshitz scaling with $z>1$. Here we generalize their analysis to arbitrary $(d+2)$-dimensional spacetime. Rather than studying the holographic superconductor, we investigate the thermodynamic and hydrodynamic properties of such black holes. We show that the black hole mass can be obtained by the first law of thermodynamics, while the other approaches for defining conserved quantities in non-relativistic backgrounds do not work. We also obtain the Gauss-Bonnet corrections to five-dimensional charged Lifshitz black holes by perturbative methods. The ratio of shear viscosity over entropy density and the DC conductivity are calculated in the presence of Gauss-Bonnet corrections.

The rest of the paper is organized as follows: We obtain the charged Lifshitz black hole solutions in general $(d+2)$ - dimensions and discuss the thermodynamics in Sec. 2. The Gauss-Bonnet corrections to such black holes in five dimensions are calculated perturbatively in Sec. 3. We compute the ratio of shear viscosity over entropy density and the DC conductivity in Sec. 4 and Sec. 5 respectively, where Gauss-Bonnet corrections are taken into account. We summarize our main results and discuss related issues in Sec. 6 .

\section{The solution and thermodynamics}

In this section we will obtain the exact solutions of charged Lifshitz black holes and discuss their thermodynamics. First we will review the charged Lifshitz black hole solution studied in [20]. The four-dimensional action (1.4) admits the following exact Lifshitz black hole solutions, whose general form can be summarized as

$$
d s^{2}=L^{2}\left[-r^{2 z} f(r)^{2} d t^{2}+\frac{g(r)^{2}}{r^{2}} d r^{2}+r^{2}\left(d \theta^{2}+\chi(\theta)^{2} d \phi^{2}\right)\right]
$$

with

$$
\begin{aligned}
& \chi(\theta)=\sin \theta \quad \text { if } \quad \mathrm{k}=1, \\
& \chi(\theta)=\theta \quad \text { if } \quad \mathrm{k}=0, \\
& \chi(\theta)=\sinh \theta \quad \text { if } \quad \mathrm{k}=-1,
\end{aligned}
$$


where $k=+1,0,-1$ corresponds to a spherical, flat, and hyperbolic horizon respectively. A $z=2$ topological black hole with a hyperbolic horizon was obtained in [13], where

$$
f(r)=\frac{1}{g(r)}=\sqrt{1-\frac{1}{2 r^{2}}} .
$$

Another solution is a $z=4$ black hole with a spherical horizon [14], where

$$
f(r)=\frac{1}{g(r)}=\sqrt{1+\frac{1}{10 r^{2}}-\frac{3}{400 r^{4}}} .
$$

At first sight, these Lifshitz black holes carry a charge that couples to the two-form field strength $F_{(2)}$, but actually they are analogous to Schwarzschild-AdS black holes rather than RN-AdS black holes. It has been shown in [20] that the system cannot undergo superconducting phase transition without additional field contents. Then a second Maxwell field $\mathcal{F}_{(2)}$ and a scalar field $\psi$ were incorporated, which were sufficient to ensure a superconducting phase transition. It should be pointed out that the scalar $\psi$ was charged under $\mathcal{F}_{(2)}$ but neutral under $F_{(2)}$ and $H_{(3)}$. The original Lifshitz gauge fields can be seen as auxiliary construction, which modify the asymptotic geometry from AdS to Lifshitz. The authors of [20] added the following term to the action

$$
S_{\mathcal{F}}=-\frac{1}{2} \int * \mathcal{F}_{(2)} \wedge \mathcal{F}_{(2)}
$$

The new $z=4$ black hole solution still takes the form of (2.1), with

$$
f(r)=\frac{1}{g(r)}=\sqrt{1+\frac{k}{10 r^{2}}-\frac{3 k^{2}}{400 r^{4}}-\frac{Q^{2}}{2 r^{4}}},
$$

where the physical charge $Q$ is induced by the second Maxwell field $\mathcal{F}_{(2)}$.

On the other hand, it has been observed in [16] that the following action

$$
S=\frac{1}{16 \pi G_{d+2}} \int d^{d+2} x \sqrt{-g}\left(R-2 \Lambda-\frac{1}{4} F^{2}-\frac{1}{2} m^{2} A^{2}\right)
$$

admits $(d+2)$-dimensional Lifshitz spacetime with arbitrary $z$

$$
d s^{2}=L^{2}\left(-r^{2 z} d t^{2}+\frac{1}{r^{2}} d r^{2}+r^{2} \sum_{i=1}^{d} d x_{i}^{2}\right)
$$

as a solution, where the values of the fields are fixed to be

$$
\Lambda=-\frac{1}{2 L^{2}}\left[z^{2}+z(d-1)+d^{2}\right], \quad A_{t}=\sqrt{\frac{2(z-1)}{z}} L r^{z}, \quad m^{2}=\frac{z d}{L^{2}} .
$$


However, it is difficult to find Lifshitz black hole solutions in general dimensions by exploring this action. Then inspired by [20], we add a second Maxwell field $\mathcal{F}_{(2)}$ to the above effective action in arbitrary dimensions and we find analytic Lifshitz black hole solutions. Consider the following action in $(d+2)$-dimensional spacetime

$$
S=\frac{1}{16 \pi G_{d+2}} \int d^{d+2} x \sqrt{-g}\left(R-2 \Lambda-\frac{1}{4} F^{2}-\frac{1}{2} m^{2} A^{2}-\frac{1}{4} \mathcal{F}^{2}\right) .
$$

The corresponding equations of motion are given as follows,

$$
\begin{aligned}
\partial_{\mu}\left(\sqrt{-g} F^{\mu \nu}\right)= & m^{2} \sqrt{-g} A^{\nu}, \quad \partial_{\mu}\left(\sqrt{-g} \mathcal{F}^{\mu \nu}\right)=0 \\
R_{\mu \nu}= & \frac{2}{d} \Lambda g_{\mu \nu}+\frac{1}{2} F_{\mu \lambda} F_{\nu}{ }^{\lambda}+\frac{1}{2} \mathcal{F}_{\mu \lambda} \mathcal{F}_{\nu}{ }^{\lambda}+\frac{1}{2} m^{2} A_{\mu} A_{\nu} \\
& -\frac{1}{4 d} F^{2} g_{\mu \nu}-\frac{1}{4 d} \mathcal{F}^{2} g_{\mu \nu} .
\end{aligned}
$$

Let us take the following ansatz for the black hole metric

$$
d s^{2}=L^{2}\left[-r^{2 z} f(r) d t^{2}+\frac{d r^{2}}{r^{2} f(r)}+r^{2} \sum_{i=1}^{d} d x_{i}^{2}\right] .
$$

It can be seen that the $t t$ and $r r$ components of the Einstein equations give

$$
R_{t}^{t}-R_{r}^{r}=-\frac{(z-1) d}{L^{2}} f(r)=-\frac{m^{2}}{2 L^{2} r^{2 z} f(r)} A_{t} A_{t}
$$

When $z=1$, the above equation leads to the following solution for the massive vector field,

$$
A_{t}=0, \quad F_{r t}=0
$$

The second Maxwell field and the cosmological constant are given by

$$
\mathcal{F}_{r t}=\frac{q L}{r^{d}}, \quad \Lambda=-\frac{d(d+1)}{2 L^{2}} .
$$

The metric of the black hole turns out to be

$$
d s^{2}=L^{2}\left[-r^{2} f(r) d t^{2}+\frac{d r^{2}}{r^{2} f(r)}+r^{2} \sum_{i=1}^{d} d x_{i}^{2}\right], \quad f(r)=1-\frac{m}{r^{d+1}}+\frac{q^{2}}{2 d(d-1) r^{2 d}} .
$$

This is simply the ordinary planar RN-AdS black hole.

When $z \neq 1$, the mass parameter and the cosmological constant remain the same as the zero-temperature Lifshitz background,

$$
m^{2}=\frac{z d}{L^{2}}, \quad \Lambda=-\frac{1}{2 L^{2}}\left[z^{2}+z(d-1)+d^{2}\right]
$$


while the massive vector field and the second Maxwell field strength are given by

$$
A_{t}=\sqrt{\frac{2(z-1)}{z}} L r^{z} f(r), \quad \mathcal{F}_{r t}=q L r^{z-d-1},
$$

where

$$
f(r)=1-\frac{q^{2}}{2 d^{2} r^{z}} .
$$

It should be emphasized that this charged Lifshitz black hole is different from the $z=1$ counterparts, as it does not admit extremal solutions. In this sense it looks more analogous to Schwarzschild-AdS black holes than RN-AdS black holes. Here the dynamical exponent $z$ cannot be arbitrary, but has to be fixed by

$$
z=2 d .
$$

One can see that in four dimensions the solution agrees with the $k=0$ black hole solution obtained in [20], after redefining the charge parameter $q$. The temperature and entropy are given by

$$
T=\frac{z}{4 \pi} r_{0}^{z}, \quad S_{\mathrm{BH}}=\frac{L^{d} V_{d}}{4 G_{d+2}} r_{0}^{d},
$$

where $r_{0}^{z} \equiv q^{2} / 2 d^{2}$ and $V_{d}$ denotes the volume of the $d$-dimensional spatial part.

Next let us calculate the mass of the Lifshitz black hole. One way is to consider the Hamiltonian approach, which was proposed in 21] and was illustrated for four-dimensional Lifshitz black holes in [14, 15]. The mass is given by the following formula

$$
M=-\frac{1}{8 \pi G_{d+2}} \int_{S_{t}^{\infty}} d^{d} x N\left(K-K_{0}\right),
$$

where $N$ is the lapse function, $K$ is the extrinsic curvature of the Lifshitz black hole metric at constant $r$ and $K_{0}$ is the corresponding extrinsic curvature for zero-temperature background solution with $f(r)=1$. One can obtain

$$
M=-\frac{V_{d} L^{d}}{8 \pi G_{d+2}} \lim _{r \rightarrow \infty} r^{3 d}(f-1) \sim r^{d},
$$

that is, the mass is divergent. However, one can still define thermodynamic mass of the solution according to the first law of black hole thermodynamics,

$$
M=\int T d S_{\mathrm{BH}}=\frac{z V_{d} L^{d}}{48 \pi G_{d+2}} r_{0}^{3 d} .
$$


Another approach was proposed in [22], where it argued that the divergences found in [14] were due to using an action which did not include the surface terms necessary to ensure that the action was finite on shell. However, if we simply generalize their analysis to general $(d+2)$-dimensional spacetime, the action can be written as

$$
\begin{aligned}
S= & S_{\text {bulk }}+S_{\text {bdy }} \\
= & \frac{1}{16 \pi G_{d+2}} \int d^{d+2} x \sqrt{-g}\left(R-2 \Lambda-\frac{1}{4} F_{\mu \nu} F^{\mu \nu}-\frac{1}{2} m^{2} A_{\mu} A^{\mu}-\frac{1}{4} \mathcal{F}^{2}\right) \\
& +\frac{1}{16 \pi G_{d+2}} \int d^{d+1} \xi \sqrt{-h}\left(2 K-\frac{2 d}{L}+f\left(A_{\alpha} A^{\alpha}\right)\right)+S_{\text {deriv }} .
\end{aligned}
$$

Here $\xi^{\alpha}$ are coordinates on the boundary, $h_{\alpha \beta}$ is the induced metric and $K_{\alpha \beta}$ is the extrinsic curvature of the boundary. $S_{\text {deriv }}$ is a collection of terms involving derivatives of the boundary fields. By fixing $f\left(A_{\alpha} A^{\alpha}\right)=-(z \alpha / L) \sqrt{-A_{\alpha} A^{\alpha}}$ and taking Dirichlet boundary condition for $\mathcal{A}_{\mu}$, one can obtain a well-defined variation of the action,

$$
\delta S=\int d^{d+1} \xi\left(s_{\alpha \beta} h^{\alpha \beta}+s_{\alpha} \delta A^{\alpha}\right)
$$

where

$$
\begin{aligned}
s_{\alpha \beta} & =\sqrt{-h}\left[\left(\pi_{\alpha \beta}+\frac{d}{L} h_{\alpha \beta}\right)+\frac{z \alpha}{2 L}\left(-A_{\alpha} A^{\alpha}\right)^{-1 / 2}\left(A_{\alpha} A_{\beta}-A_{\gamma} A^{\gamma} h_{\alpha \beta}\right)\right]+s_{\alpha \beta}^{\text {deriv }}, \\
s_{\alpha} & =-\sqrt{-h}\left(n^{\mu} F_{\mu \alpha}-\frac{z \alpha}{L}\left(-A_{\alpha} A^{\alpha}\right)^{-1 / 2} A_{\alpha}\right)+s_{\alpha}^{\text {deriv }}
\end{aligned}
$$

and $\pi_{\alpha \beta} \equiv K_{\alpha \beta}-K h_{\alpha \beta}$.

The contribution of the second Maxwell field $\mathcal{F}_{\mu \nu}$ to the on-shell action can be estimated as

$$
\int \sqrt{-g} \mathcal{F}^{2} \sim \int r^{d-1}
$$

which is divergent. According to [22, for asymptotically Lifshitz spacetimes, the energy is given by

$$
\mathcal{E}=2 s^{t}{ }_{t}-s^{t} A_{t}
$$

Substituting the black hole solution into (2.27), the result turns out to be

$$
\mathcal{E}=\lim _{r \rightarrow \infty} d L r^{3 d}\left(f(r)-f(r)^{1 / 2}\right) \sim r^{d} \rightarrow \infty
$$

The divergence implies that the boundary terms in (2.25) are a priori insufficient to render the on-shell action finite. We expect to remove the divergences in further investigations on the definitions of conserved quantities in asymptotically Lifshitz spacetimes. 


\section{Gauss-Bonnet corrections}

In this section we study Gauss-Bonnet corrections to five-dimensional charged Lifshitz black holes. Generally speaking, it is always difficult to obtain exact black hole solutions in higher derivative gravity, so we will try to find black hole solutions in Gauss-Bonnet gravity by perturbative methods, following [23].

First we rewrite the five-dimensional charged Lifshitz black hole solution as follows,

$$
\begin{aligned}
& d s^{2}=L^{2}\left[-r^{2 z} f(r) d t^{2}+\frac{d r^{2}}{r^{2} f(r)}+r^{2}\left(d x_{1}^{2}+d x_{2}^{2}+d x_{3}^{2}\right)\right], \quad f(r)=1-\frac{q^{2}}{18 r^{z}} \equiv 1-\frac{r_{0}^{z}}{r^{z}} \\
& z=2 d=6, \quad m^{2}=\frac{18}{L^{2}}, \quad \Lambda=-\frac{1}{2 L^{2}}\left[z^{2}+(d-1) z+d^{2}\right]=-\frac{57}{2 L^{2}} \\
& A_{t}=\sqrt{\frac{5}{3}} r^{z} f(r), \quad \mathcal{F}_{r t}=q L r^{2} .
\end{aligned}
$$

Now consider the following effective action containing Gauss-Bonnet corrections

$$
\begin{aligned}
S= & \frac{1}{16 \pi G_{5}} \int d^{5} x \sqrt{-g}\left[R-2 \Lambda-\frac{1}{4} F_{\mu \nu} F^{\mu \nu}-\frac{1}{2} m^{2} A_{\mu} A^{\mu}-\frac{1}{4} \mathcal{F}_{\mu \nu} \mathcal{F}^{\mu \nu}\right. \\
& \left.+\frac{\lambda_{\mathrm{GB}}}{2} L^{2}\left(R_{\mu \nu \lambda \delta} R^{\mu \nu \lambda \delta}-4 R_{\mu \nu} R^{\mu \nu}+R^{2}\right)\right] .
\end{aligned}
$$

The equations of motion for the gauge fields remain the same as before,

$$
\partial_{\mu}\left(\sqrt{-g} F^{\mu \nu}\right)=m^{2} \sqrt{-g} A^{\nu}, \quad \partial_{\mu}\left(\sqrt{-g} \mathcal{F}^{\mu \nu}\right)=0,
$$

while the Einstein equations take the following form

$$
R_{\mu \nu}-\frac{1}{2} R g_{\mu \nu}=-\Lambda g_{\mu \nu}+T_{\mu \nu}^{\mathrm{M}}+T_{\mu \nu}^{\mathrm{R}},
$$

with

$$
\begin{aligned}
T_{\mu \nu}^{\mathrm{M}}= & \frac{1}{2} F_{\mu \lambda} F_{\nu}{ }^{\lambda}+\frac{1}{2} m^{2} A_{\mu} A_{\nu}+\frac{1}{2} \mathcal{F}_{\mu \lambda} \mathcal{F}_{\nu}{ }^{\lambda}-\frac{1}{8} F^{2} g_{\mu \nu}-\frac{1}{4} m^{2} A^{2} g_{\mu \nu}-\frac{1}{8} \mathcal{F}^{2} g_{\mu \nu}, \\
T_{\mu \nu}^{\mathrm{R}}= & \frac{\lambda_{\mathrm{GB}}}{2} L^{2}\left[\frac{1}{2} g_{\mu \nu}\left(R_{\mu \nu \lambda \delta} R^{\mu \nu \lambda \delta}-4 R_{\mu \nu} R^{\mu \nu}+R^{2}\right)\right. \\
& \left.-2 R R_{\mu \nu}+4 R_{\mu \gamma} R^{\gamma}{ }_{\nu}+4 R^{\gamma \delta} R_{\gamma \mu \delta \nu}-2 R_{\mu \gamma \delta \lambda} R_{\nu}{ }^{\gamma \delta \lambda}\right] .
\end{aligned}
$$

The Einstein equations can be recasted as

$$
R_{\mu \nu}=\frac{2}{3} \Lambda g_{\mu \nu}+T_{\mu \nu}^{\mathrm{M}}+T_{\mu \nu}^{\mathrm{R}}-\frac{1}{3}\left(T^{\mathrm{M}}+T^{\mathrm{R}}\right) g_{\mu \nu},
$$


where $T^{\mathrm{M}} \equiv g^{\mu \nu} T_{\mu \nu}^{\mathrm{M}}$ and $T^{\mathrm{R}} \equiv g^{\mu \nu} T_{\mu \nu}^{\mathrm{R}}$.

The ansatz for the metric is given by

$$
d s^{2}=\frac{L^{2}}{\rho^{2}}\left[-e^{2 A(\rho)} d t^{2}+e^{-2 B(\rho)} d \rho^{2}+d x_{1}^{2}+d x_{2}^{2}+d x_{3}^{2}\right] .
$$

One can derive the following relations for the components of the Ricci tensor

$$
\begin{aligned}
R_{t}^{t}-R_{\rho}^{\rho} & =\frac{3}{L^{2}} e^{2 B(\rho)} \rho\left(A^{\prime}(\rho)-B^{\prime}(\rho)\right), \\
\frac{1}{3}\left(R_{t}^{t}-R_{\rho}^{\rho}\right)-R_{1}^{1} & =-\frac{1}{L^{2}}\left(\frac{e^{2 B(\rho)}}{\rho^{4}}\right)^{\prime} \rho^{5},
\end{aligned}
$$

where the prime stands for derivative with respect to $\rho$. To obtain the perturbative solution, we will substitute the field configurations of the unperturbed solution into $T_{\mu \nu}^{\mathrm{M}}$ and $T_{\mu \nu}^{\mathrm{R}}$. By combining (3.6) and (3.8), we can obtain

$$
-\frac{1}{L^{2}}\left(\frac{e^{2 B(\rho)}}{\rho^{4}}\right)^{\prime} \rho^{5}=\frac{2}{3} T_{t}^{\mathrm{M}^{t}}+\frac{2}{3} T_{t}^{\mathrm{R}^{t}}-\frac{2}{3} \Lambda .
$$

Then the function $e^{2 B(\rho)}$ reads

$$
e^{2 B(\rho)}=f(\rho)\left(1+\lambda_{\mathrm{GB}} f(\rho)\right), \quad f(\rho)=1-\frac{q^{2}}{18} \rho^{6} .
$$

From the first equation of (3.8), we can obtain

$$
A(\rho)=B(\rho)+\frac{L^{2}}{3} \int \frac{1}{\rho} e^{2 B(\rho)}\left(R_{t}^{t}-R_{\rho}^{\rho}\right) .
$$

After substituting the unperturbed solution, the function $e^{2 A(\rho)}$ is given by

$$
e^{2 A(\rho)}=e^{2 B(\rho)} \rho^{-\left(z_{0}-1\right)\left(2+4 \lambda_{\mathrm{GB}}\right)} \exp \left[\frac{2\left(z_{0}-1\right)}{z_{0}} \frac{\lambda_{\mathrm{GB}} q^{2}}{d^{2}} \rho^{z_{0}}\right],
$$

where $z_{0}=2 d=6$ denotes the dynamical exponent of the unperturbed solution.

Finally, performing the coordinate transformation $\rho=1 / r$, the perturbed solution in Gauss-Bonnet gravity can be summarized as follows

$$
d s^{2}=L^{2}\left[-f(r) h(r) r^{2 z} d t^{2}+\frac{d r^{2}}{r^{2} f(r)}+r^{2}\left(d x_{1}^{2}+d x_{2}^{2}+d x_{3}^{2}\right)\right],
$$

where

$$
\begin{aligned}
f(r) & =f_{0}(r)\left(1+\lambda_{\mathrm{GB}} f_{0}(r)\right), \quad f_{0}(r)=1-\frac{q^{2}}{18 r^{6}}, \\
h(r) & =\exp \left[\frac{5 \lambda_{\mathrm{GB}} q^{2}}{27 r^{6}}\right],
\end{aligned}
$$


and

$$
z=z_{0}+2 \lambda_{\mathrm{GB}}\left(z_{0}-1\right), \quad z_{0}=2 d=6
$$

The first-order solutions of the gauge fields can also be obtained by substituting the perturbed metric into the equations of motion (3.3), where we have made the following ansatz

$$
A_{t}=\sqrt{\frac{5}{3}} r^{z_{0}} f_{0}(r)+\lambda_{\mathrm{GB}} A_{1 t}(r), \quad \mathcal{F}_{r t}=q L r^{2}+\lambda_{\mathrm{GB}} \mathcal{F}_{1 r t} .
$$

The first-order corrections are given by

$$
\begin{aligned}
A_{1 t}= & c_{1}\left(r^{6}-r_{0}^{6}\right)+c_{2}\left(-\frac{r^{3}}{6 r_{0}^{6}}+\frac{\operatorname{arctanh}\left(\frac{r^{3}}{r_{0}^{3}}\right)}{6 r_{0}^{9}}\left(r^{6}-r_{0}^{6}\right)\right) \\
& +\mathcal{O}\left(\log \left(r^{6}-r_{0}^{6}\right)\right), \\
\mathcal{F}_{1 r t}= & \frac{5}{3} q L \frac{r_{0}^{6}}{r^{4}} .
\end{aligned}
$$

Here are some remarks on the perturbed black hole solution:

- The horizon still locates at $r=r_{0}$ for the perturbed black hole solution.

- The temperature and entropy are given by

$$
T=\frac{1}{4 \pi} z_{0} r_{0}^{z} \exp \left(\frac{5}{3} \lambda_{\mathrm{GB}}\right), \quad S_{\mathrm{BH}}=\frac{1}{4 G_{5}} r_{0}^{3} L^{3} V_{3} .
$$

Note that the Bekenstein-Hawking formula still holds for planar black holes in Gauss-Bonnet gravity.

- The $1 / N$ effects in non-relativistic gauge-gravity duality were investigated extensively in [24], where they argued that the dynamical exponent would be renormalized in higher derivative gravity except for $z_{0}=1$. Here our results support their arument.

\section{Calculating $\eta / s$}

The AdS/CFT correspondence has provided us a powerful tool for investigating the hydrodynamic properties of strongly coupled field theories. One remarkable progress is the calculation of shear viscosity in the dual gravity side. It has been found that the ratio of 
shear viscosity over entropy density is $1 / 4 \pi$ for a large class of CFTs with Einstein gravity duals in the large $\mathrm{N}$ limit. Therefore, it was conjectured that $1 / 4 \pi$ is a universal lower bound for all materials, which is the so-called Kovtun-Son-Starinets(KSS) bound [25]. However, in [23, 26, 27] it was observed that in $R^{2}$ gravity such a lower bound was violated and a new lower bound $4 / 25 \pi$ was proposed by considering the causality of the dual field theory. Corrections to $\eta / s$ for various examples were nicely investigated in [28, 29]. For more developments, see e.g. [30].

It was conjectured in [31] that the shear viscosity is fully determined by the effective coupling of the transverse gravitons on the horizon in the dual gravity description. This was confirmed in [32] via the scalar membrane paradigm and in [33] by calculating the on-shell action of the transverse gravitons. However, the latter formalism is not covariant under coordinate transformations, then the choice of coordinate system of the background black hole geometry affects the form of the action of the transverse gravitons. A new formalism was proposed in [34], where a three-dimensional effective metric $\tilde{g}_{\mu \nu}$ was introduced and the transverse gravitons were minimally coupled to this new effective metric. The action in this new formalism can take a covariant form. Similar discussions on this issue were also presented in [35].

The shear viscosity is given by the Kubo formula

$$
\eta=\lim _{\omega \rightarrow 0} \frac{1}{2 \omega i}\left(G_{x_{1} x_{2}, x_{1} x_{2}}^{A}(\omega, 0)-G_{x_{1} x_{2}, x_{1} x_{2}}^{R}(\omega, 0)\right),
$$

where the retarded Green's function $G_{\mu \nu, \lambda \rho}^{R}$ is defined by

$$
G_{\mu \nu, \lambda \rho}^{R}=-i \int d^{4} x e^{-i k \cdot x} \theta(t)<\left[T_{\mu \nu}(x), T_{\lambda \rho}(0)\right]>,
$$

and the advanced Green's function satisfies $G_{\mu \nu, \lambda \rho}^{A}(k)=G_{\mu \nu, \lambda \rho}^{R}(k)^{*}$. According to the fieldoperator correspondence, such Green's functions can be calculated through the effective action of the gravitons of the dual gravity theory.

Consider tensor perturbation $h_{12}=h_{12}(t, u, z)$, where $u$ is the radial coordinate and the momentum of the perturbation points along the $x_{3} \equiv z$ axis. Then we denote $\phi=h_{2}^{1}$ and write $\phi$ as $\phi(t, u, z)=\phi(u) e^{-i \omega t+i p z}$. If the transverse gravitons can be decoupled from other perturbations, the effective bulk action of the transverse gravitons can be written in a general form

$$
S=\frac{V_{1,2}}{16 \pi G}\left(-\frac{1}{2}\right) \int d^{3} x \sqrt{-\tilde{g}}\left(\tilde{K}(u) \tilde{g}^{M N} \tilde{\nabla}_{M} \phi \tilde{\nabla}_{N} \phi+m^{2} \phi^{2}\right)
$$


up to some total derivatives. Here $\tilde{g}_{M N}, M, N=t, u, z$ is a three-dimensional effective metric, $m$ is an effective mass and $\tilde{\nabla}_{M}$ is the covariant derivative using $\tilde{g}_{M N}$. Notice that $\phi$ is a scalar in the three dimensions $t, u, z$, while it is not a scalar in the whole five dimensions. As we have assumed that $\phi=h_{2}^{1}(t, u, z)$, the effective action for $\phi$ can be seen as a deduced three-dimensional action where the other two irrelevant directions can be integrated out. In this sense it is not the usual dimensional reduction. The three-dimensional effective action itself is general covariant and $\tilde{K}(u)$ is a scalar under general coordinate transformations. In the following we will use $g_{\mu \nu}$ to denote the whole five-dimensional background.

By performing the coordinate transformation $u=r_{0}^{3} / r^{3}$, the perturbative black hole solution in Gauss-Bonnet gravity (3.13) can be rewritten as

$$
d s^{2}=L^{2}\left[-(1-u) F(u) d t^{2}+\frac{d u^{2}}{(1-u) G(u)}+\frac{r_{0}^{2}}{u^{2 / 3}}\left(d x_{1}^{2}+d x_{2}^{2}+d x_{3}^{2}\right)\right],
$$

where

$$
\begin{aligned}
& F(u)=(1+u)\left(1+\lambda_{\mathrm{GB}}\left(1-u^{2}\right)\right) h(u) r_{0}^{2 z} u^{-2 z / 3}, \\
& h(u)=\exp \left[\frac{5}{27} \lambda_{\mathrm{GB}} u^{2}\right], \quad G(u)=9(1+u)\left(1+\lambda_{\mathrm{GB}}\left(1-u^{2}\right)\right) u^{2}, \\
& z=z_{0}+2 \lambda_{\mathrm{GB}}\left(z_{0}-1\right), \quad z_{0}=2 d=6 .
\end{aligned}
$$

Notice that here the horizon locates at $u=1$. Following [34], we write down the action of the transverse gravitons in momentum space

$$
\begin{aligned}
S= & \frac{V_{1,2}}{16 \pi G}\left(-\frac{1}{2}\right) \int \frac{d w d p}{(2 \pi)^{2}} d u \sqrt{-\tilde{g}}\left[\tilde { K } ( u ) \left(\tilde{g}^{u u} \phi^{\prime} \phi^{\prime}\right.\right. \\
& \left.\left.+w^{2} \tilde{g}^{t t} \phi^{2}+p^{2} \tilde{g}^{z z} \phi^{2}\right)+m^{2} \phi^{2}\right],
\end{aligned}
$$

where

$$
\begin{aligned}
& \phi(t, u, z)=\int \frac{d w d p}{(2 \pi)^{2}} \phi(u ; k) e^{-i w t+i p z}, \\
& k=(w, 0,0, p), \quad \phi(u ;-k)=\phi^{*}(u ; k),
\end{aligned}
$$

and the prime denotes derivative with respect to $u$. The subsequent steps are similar to those exhibited in [34], which will not be shown explicitly. We can find that here we still have the following formula for $\eta$

$$
\eta=\left.\frac{1}{16 \pi G}\left(\sqrt{\tilde{g_{z z}}} \tilde{K}(u)\right)\right|_{u=1} .
$$


Next we calculate the effective action of the transverse gravitons in the background (4.4). By checking directly from the first-order Einstein equations it can be found that the transverse gravitons can get decoupled from other perturbations. Then the effective action of the transverse gravitons can be obtained by keeping the quadratic terms of $\phi$ in the original action and it can be written in the form of (4.3) with the effective three-dimensional metric

$$
\begin{gathered}
\tilde{g}^{u u}=\left(1+\frac{\lambda_{\mathrm{GB}}}{2} \frac{A g_{t t}^{\prime} g^{u u}}{u g_{t t}}\right) g^{u u}, \\
\tilde{g}^{t t}=\left[1+\frac{\lambda_{\mathrm{GB}}}{2}\left(\frac{A g^{\prime u u}}{u}-\frac{\left(A^{2}+2 A\right) g^{u u}}{u^{2}}\right)\right] g^{t t}, \\
\tilde{g}^{z z}=\left[1+\frac{\lambda_{\mathrm{GB}}}{2}\left(\frac{g_{t t}^{\prime 2} g^{u u}}{g_{t t}^{2}}-\frac{g_{t t}^{\prime} g^{\prime u u}}{g_{t t}}-\frac{2 g^{u u} g_{t t}^{\prime \prime}}{g_{t t}}\right)\right] g^{z z},
\end{gathered}
$$

where $A \equiv 4 / z_{0}=2 / 3$.

In fact, the effective action of the transverse gravitons can also be written as

$$
S=\frac{1}{16 \pi G}\left(-\frac{1}{2}\right) \int d^{5} x \sqrt{-g} \tilde{g}^{\mu \nu} \partial_{\mu} \phi \partial_{\nu} \phi
$$

where $\tilde{g}^{\mu \nu}=\tilde{g}^{\mu \nu}$ for $\mu, \nu=t, u, z$ and $\tilde{g}^{\mu \nu}=g^{\mu \nu}$ for $\mu, \nu=x_{1}, x_{2}$. Then $\tilde{K}(u)=$ $\sqrt{-g} / \sqrt{-\tilde{g}}$. Finally, by substituting (4.11) into (4.8) and recalling the fact that the Bekenstein-Hawking area law still holds for planar black holes in Gauss-Bonnet gravity, we have

$$
\begin{aligned}
\frac{\eta}{s} & =\frac{1}{4 \pi}\left[1-\frac{A}{2} \lambda_{\mathrm{GB}} G(1)\right] \\
& =\frac{1}{4 \pi}\left(1-6 \lambda_{\mathrm{GB}}\right) .
\end{aligned}
$$

Here are some remarks on this result:

- The Gauss-Bonnet corrections to asymptotically Lifshitz black holes and the higher order corrections to $\eta / s$ were calculated in [36], where the result turned out to be

$$
\frac{\eta}{s}=\frac{1}{4 \pi}\left[1-\left(z_{0}+3\right) \lambda_{\mathrm{GB}}\right]
$$

It can be seen that the above expression reduces to the result obtained in [26] when $z_{0}=1$. However, here we cannot reproduce the same result as the dynamical exponent $z_{0}$ is not a free parameter but is fixed by the number of spatial coordinates. 
- $\lambda_{\mathrm{GB}}$ should have an upper bound $1 / 6$ to ensure a non-vanishing $\eta / s$. The upper bound of $\lambda_{\mathrm{GB}}$ was discussed in [37] where it was found to be $1 / 4$ by the constraints of causality and stability. Here a similar upper bound in non-relativistic theory requires further understanding. As the dual field theory is non-relativistic, causality cannot be served as a constraint but stability may still work.

- In the literatures discussing the ratio of shear viscosity over entropy density in higher derivative theory of gravity, the new lower bound of $\eta / s^{-4} / 25 \pi$-can be obtained by considering the causality of the boundary field theory. However, here we cannot take such a constraint as the dual field theory is non-relativistic, which is similar to the case discussed in [36]. However, we expect that the issue of stability may still exert some constraint on $\eta / s$.

\section{Conductivity}

In this section we calculate the DC conductivity of the Lifshitz black holes. Firstly, in order to obtain a well-defined result of the 'electrical' conductivity, we imagine coupling the CFT current to an external or auxiliary vector field, according to [38, 39]. The global $U(1)$ symmetry of the theory is gauged with a small coupling $e$. To leading order in $e$, the effects of the auxiliary gauge field can be neglected and the conductivity can be determined from the original CFT. Then the DC conductivity is given by

$$
\sigma=-\lim _{\omega \rightarrow 0} \frac{e^{2}}{\omega} \operatorname{Im} G_{x, x}^{R}(\omega, \mathbf{k}=0)
$$

where the retarded correlation function of the global $U(1)$ symmetry currents is given by a Kubo formula similar to the one for shear viscosity,

$$
G_{x, x}^{R}(\omega, \mathbf{k}=0)=-i \int d t d \mathbf{x} e^{i \omega t} \theta(t)<\left[J_{x}(x), J_{x}(0)\right]>.
$$

Due to the existence of the non-vanishing component $\mathcal{A}_{t}$ of the bulk gauge field in the background, the perturbation of the gauge field $\mathcal{A}_{x}$ gets coupled to the shear mode graviton, i.e., metric perturbations of the form $h_{x i}$. However, the contribution of $h_{x i}$ can be integrated out by imposing gauge invariance upon the two sets of perturbations. Then we can obtain an action that only contains the $\mathcal{A}_{x}$ fluctuation. Therefore, the most convenient way to perform the calculations is the effective action approach. Such an approach 
was applied in [40], where the conductivity for five-dimensional charged planar AdS black holes was computed in the presence of a general set of four-derivative interactions. We will follow their approach to calculate the conductivity of the charged Lifshitz black holes. The perturbations of the metric and the gauge field are given by

$$
\begin{aligned}
h_{t}{ }^{x} & =\int \frac{d^{4} k}{(2 \pi)^{4}} t_{k}(u) e^{-i \omega t+i k z}, \\
h_{u}{ }^{x} & =\int \frac{d^{4} k}{(2 \pi)^{4}} h_{k}(u) e^{-i \omega t+i k z}, \\
\mathcal{A}_{x} & =\int \frac{d^{4} k}{(2 \pi)^{4}} a_{k}(u) e^{-i \omega t+i k z},
\end{aligned}
$$

where we have denoted $x_{1} \equiv x$ and $x_{3} \equiv z$. Let us consider the leading order solution first. After performing coordinate transformation $u=r_{0}^{3} / r^{3}$, the metric turns out to be

$$
d s^{2}=L^{2}\left[-\frac{r_{0}^{12}}{u^{4}} f_{0}(u) d t^{2}+\frac{d u^{2}}{9 u^{2} f_{0}(u)}+\frac{r_{0}^{2}}{u^{2 / 3}}\left(d x_{1}^{2}+d x_{2}^{2}+d x_{3}^{2}\right)\right],
$$

where $f_{0}(u)=1-u^{2}$. The horizon locates at $u=u_{0}=1$. As a gauge choice, we would like to set the perturbation $h_{u}{ }^{x}$ to be zero. By comparing the $x x$ and $u x$ components of the Einstein equations, we can arrive at the following constraint

$$
g_{x x} t_{k}^{\prime}=-\mathcal{A}_{t}^{\prime} a_{k}
$$

which is the same as that in [40].

After taking the above constraint and the gauge choice $h_{k}(u)=0$, the quadratic action for $a_{k}$ takes the following form

$$
\tilde{I}_{a}^{(2)}=\frac{1}{16 \pi G_{5}} \int \frac{d^{4} k}{(2 \pi)^{4}} d u\left(N(u) a_{k}^{\prime} a_{-k}^{\prime}+M(u) a_{k} a_{-k}\right)
$$

where

$$
N(u)=-3 L r_{0}^{7} u^{-4 / 3} f_{0}(u), \quad M(u)=\frac{L \omega^{2} u^{2 / 3}}{3 r_{0}^{5} f_{0}(u)}-\frac{3 u^{8 / 3}}{L r_{0}^{5}} \mathcal{A}_{t}^{\prime 2} .
$$

Such an effective action can either be obtained by expanding the original action up to quadratic order of $a_{k}$, or by directly considering the Maxwell equation of the perturbations. The equation of motion for $a_{k}$ can be re-expressed as

$$
\partial_{u} j_{k}(u)=\frac{1}{8 \pi G_{5}} M(u) a_{k}(u)
$$


where

$$
j_{k}(u) \equiv \frac{\delta \tilde{I}_{a}^{(2)}}{\delta a_{-k}^{\prime}}=\frac{1}{8 \pi G_{5}} N(u) a_{k}^{\prime}(u) .
$$

In the near horizon region, the equation of motion can be solved by taking the ansatz

$$
a_{k}(u)=C f_{0}(u)^{\beta}
$$

It turns out that by requiring regularity at the horizon, we still have $\beta= \pm i \omega / 4 \pi T$ as usual. The infalling boundary condition fixes $\beta=-i \omega / 4 \pi T$.

Next, rather than solving the equation of motion for $a_{k}$ as in the conventional cases, we follow the prescription described in [40]. According to [32], the condition of regularity at the horizon $u=u_{0}$ requires

$$
j_{k}\left(u_{0}\right)=-i \omega \lim _{u \rightarrow u_{0}} \frac{N(u)}{8 \pi G_{5}} \sqrt{-\frac{g_{u u}}{g_{t t}}} a_{k}\left(u_{0}\right)+\mathcal{O}\left(\omega^{2}\right),
$$

where we are expanding in small $\omega$ with the zero-frequency limit of (5.1) in mind. The flux factor can be identified by evaluating the on-shell action, which simply yields

$$
2 \mathcal{F}_{k}=j_{k}(u) a_{-k}(u)
$$

Then one can obtain the Green's function (5.2) by evaluating the flux factor at the asymptotic boundary. The DC conductivity is given by a formula which is analogous to the one for shear viscosity

$$
\sigma=\lim _{u, \omega \rightarrow 0} \frac{e^{2}}{\omega} \operatorname{Im}\left[\frac{2 \mathcal{F}_{k}}{a_{k}(u) a_{-k}(u)}\right]_{\mathbf{k}=0}=\left.e^{2} \lim _{u, \omega \rightarrow 0} \frac{\operatorname{Im}\left[j_{k}(u) a_{-k}(u)\right]}{\omega a_{k}(u) a_{-k}(u)}\right|_{\mathbf{k}=0} .
$$

In the computations of shear viscosity via the membrane paradigm [32, the evolution of the canonical momentum in the radial direction is completely trivial in the low-frequency limit. Then it can be evaluated at any radial position and it is calculated at the horizon. However, here neither $j_{k}$ nor $\omega a_{k}$ has a trivial evolution along the radial direction even in the low-frequency limit. It can be seen from the equation of motion that the effective mass $M(u)$ no longer vanishes in the low-frequency limit due to the existence of $\mathcal{A}_{t}^{\prime}$, so the equation of motion still produces a nontrivial flow along the radial direction. However, it was observed in [40] that the radial evolution of the numerator of (5.12) is trivial,

$$
\partial_{u} \operatorname{Im}\left[j_{k}(u) a_{-k}(u)\right]=\operatorname{Im}\left[f_{1}(u) a_{k} a_{-k}+f_{2}(u) j_{k} j_{-k}\right]=0,
$$


which is independent of taking the low-frequency limit. Therefore we can evaluate the numerator at the horizon. Moreover, $j_{k}(u)$ should satisfy the regularity condition given in (5.10) at the horizon. The conductivity can be expressed as

$$
\sigma=\left.\frac{e^{2}}{8 \pi G_{5}} \kappa_{2}^{A}\left(u_{0}\right) \frac{\mathcal{N}\left(u_{0}\right)}{\mathcal{N}(0)}\right|_{\mathbf{k}=0}
$$

where

$$
\kappa_{2}^{A}(u)=-N(u) \sqrt{-\frac{g_{u u}}{g_{t t}}}=L r_{0} u^{-1 / 3}, \quad \mathcal{N}(u)=a_{k}(u) a_{-k}(u)
$$

As emphasized in [40], $\mathcal{N}(u)$ is real and so independent of $\omega$ up to $\mathcal{O}\left(\omega^{2}\right)$. It also means that at this order $\mathcal{N}(u)$ is completely regular at the horizon. Then we can solve $a_{k}(u)$ by imposing regularity at the horizon and setting $\omega$ to zero. Thus the equation of motion can be simplified

$$
\partial_{u}\left(u^{-4 / 3} f_{0}(u) a_{k}^{\prime}(u)\right)=2 u^{-4 / 3} a_{k}(u)
$$

whose solution is given by

$$
a_{k}(u)=a_{k}(0)_{2} F_{1}\left[-\frac{1+i \sqrt{71}}{12},-\frac{1-i \sqrt{71}}{12},-\frac{1}{6}, u^{2}\right] .
$$

The approximate solution turns out to be

$$
a_{k}(u)=a_{0}\left(1-3 u^{2}\right)
$$

Finally, in the leading order background, the conductivity is

$$
\sigma=\frac{e^{2}}{2 \pi G_{5}} L r_{0}
$$

Recall that the temperature is given by $T=z_{0} r^{z_{0}} / 4 \pi$, the expression for the conductivity can be rewritten as

$$
\sigma=\frac{e^{2}}{2 \pi G_{5}}\left(\frac{\pi}{z_{0}}\right)^{\frac{1}{z_{0}}} T^{\frac{1}{z_{0}}}
$$

We can see that when $z_{0}=1$ (although $z_{0}=6$ is fixed here), the above result reproduces the conventional relation $\sigma \sim T$. However, the physical meaning of the non-trivial dependence on $z_{0}$ in the relation between $\sigma$ and $T$ requires further interpretation.

The calculations in the first-order background are straightforward. The perturbative black 
hole solution in Gauss-Bonnet gravity reads

$$
\begin{aligned}
& d s^{2}=L^{2}\left[-(1-u) F(u) d t^{2}+\frac{d u^{2}}{(1-u) G(u)}+\frac{r_{0}^{2}}{u^{2 / 3}}\left(d x_{1}^{2}+d x_{2}^{2}+d x_{3}^{2}\right)\right], \\
& F(u)=(1+u)\left(1+\lambda_{\mathrm{GB}}\left(1-u^{2}\right)\right) h(u) r_{0}^{2 z} u^{-2 z / 3} \\
& h(u)=\exp \left[\frac{5}{27} \lambda_{\mathrm{GB}} u^{2}\right], \quad G(u)=9(1+u)\left(1+\lambda_{\mathrm{GB}}\left(1-u^{2}\right)\right) u^{2}, \\
& z=z_{0}+2 \lambda_{\mathrm{GB}}\left(z_{0}-1\right), \quad z_{0}=2 d=6
\end{aligned}
$$

and the perturbations are given by (5.3) . We still expand the action up to quadratic terms for $a_{k}, t_{k}, h_{k}$ and obtain a constraint by setting $h_{k}=0$. Plugging this constraint back into the action and keeping terms linear in $\lambda_{\mathrm{GB}}$ we can obtain an action of the form (5.6). Here the equation of motion for $a_{k}$ becomes much more complicated and it is quite difficult to obtain an analytic result. Then we solve the equation of motion up to leading order of $\lambda_{\mathrm{GB}}$. The final result is given by

$$
\sigma=\frac{e^{2}}{2 \pi G_{5}} L r_{0}\left(1+\frac{5}{3} \lambda_{\mathrm{GB}}\right)
$$

\section{Summary and discussion}

We study exact solutions of charged Lifshitz black holes and the corresponding thermodynamic and hydrodynamic properties in this paper. We generalize the four-dimensional solution obtained in [20] to arbitrary $(d+2)$-dimensional cases by adding a second Maxwell field in the effective action. The black hole solutions we find exhibit an unusual thermodynamic behavior. We cannot obtain a finite mass via the Hamiltonian approach [21] or the recently proposed prescriptions for non-relativistic backgrounds [14, 15, 22]. However, the mass of the black hole can still be obtained by the first law of thermodynamics. We also obtain the five-dimensional black hole solutions in Gauss-Bonnet gravity by perturbative methods. Furthermore, we calculate the ratio of shear viscosity over entropy density and the DC conductivity in the presence of Gauss-Bonnet corrections.

Here the ratio of shear viscosity over entropy density in Gauss-Bonnet gravity also violates the conjectured KSS bound, which is similar to the known examples. However, unlike the case studied in [36], here we cannot reproduce the result for planar AdS black holes in Gauss-Bonnet gravity, as the dynamical exponent $z_{0}$ is not arbitrary but fixed by the number of the spatial coordinates. In relativistic cases, a new lower bound can be obtained 
by taking the causality of the dual boundary field theory as a constraint. However, for the non-relativistic cases, the causality of the boundary field theory cannot be taken as a constraint, as the speed of light tends to infinity. We expect that some other criterions which are valid both in relativistic and non-relativistic backgrounds, such as stability, unitarity and locality, may introduce a new lower bound for non-relativistic cases.

When we rewrite the conductivity as a function of the temperature $T$, it shows that the conductivity is proportional to a non-trivial power of $T$, where the power is determined by $z_{0}$. The dependence on $z_{0}$ requires further understanding, although it reduces to the conventional case $\sigma \sim T$ when $z_{0}=1$.

The definition of the conductivity takes a slightly different form in [40],

$$
\sigma=-\lim _{\omega \rightarrow 0} \frac{e^{2} L_{*}^{2}}{\omega} \operatorname{Im} G_{x, x}^{R}(\omega, \mathbf{k}=0)
$$

where $L_{*}$ is some scale to make sure that the chemical potential has the appropriate unit of energy after rescaling $\mathcal{A}_{\mu}=L_{*} \tilde{\mathcal{A}}_{\mu}$. The ratio $\sigma T^{2} / \eta e^{2}$ was also examined in [40]. If we take this definition and fix $L_{*}=\pi L$ as they did, the ratio is given by

$$
\frac{\sigma T^{2}}{\eta e^{2}}=\frac{1}{2} z_{0}^{2} r^{2 z_{0}-2} \text {. }
$$

It still has a non-trivial dependence on $z_{0}$. When $z_{0}=1$, this ratio is $1 / 2$, which seems to satisfy the upper bound $\sigma T^{2} / \eta e^{2}=1$. However, as pointed out in [39], the definition of $\sigma$ (or $\eta$ ) involves an arbitrary choice of normalization for the corresponding current. So in order to find a universal bound on conductivity, it is more natural to incorporate a quantity which is independent of the normalization.

Recently there have been several interesting papers on the transport coefficients for extremal black holes in higher derivative gravity, e.g. [41, 42, 43, 44, 45]. One conclusion is that for extremal black holes the DC conductivity is always zero, even in higher derivative gravity theories. The near horizon geometry of extremal black holes contains an $A d S_{2}$ part, which plays an important role in the calculations. Here the DC conductivity is also zero if we take $T=0$. However, the behavior of charged Lifshitz black holes is more analogous to Schwarzschild-AdS black holes rather than RN-AdS black holes thus we cannot have $A d S_{2}$ near horizon geometry. We believe that for "real" extremal charged Lifshitz black holes, the conclusion will be the same as the relativistic counterparts, as they will exhibit similar near horizon structure. 


\section{Acknowledgements}

We thank Rong-Gen Cai for collaboration at the initial stage of this work and for valuable comments on the manuscript. Part of this work was done during DWP's visit to Department of Physics, National Central University and we would like to thank ChiangMei Chen for warm hospitality and helpful discussion. This work was supported by the Korea Science and Engineering Foundation(KOSEF) grant funded by the Korea government(MEST) through the Center for Quantum Spacetime(CQUeST) of Sogang University with grant number R11-2005-021.

\section{References}

[1] J. M. Maldacena, "The large N limit of superconformal field theories and supergravity," Adv. Theor. Math. Phys. 2, 231 (1998) [Int. J. Theor. Phys. 38, 1113 (1999)] arXiv:hep-th/9711200|.

[2] S. S. Gubser, I. R. Klebanov and A. M. Polyakov, "Gauge theory correlators from non-critical string theory," Phys. Lett. B 428, 105 (1998) arXiv:hep-th/9802109.

[3] E. Witten, "Anti-de Sitter space and holography," Adv. Theor. Math. Phys. 2, 253 (1998) arXiv:hep-th/9802150.

[4] O. Aharony, S. S. Gubser, J. M. Maldacena, H. Ooguri and Y. Oz, "Large N field theories, string theory and gravity," Phys. Rept. 323, 183 (2000) arXiv:hep-th/9905111.

[5] S. A. Hartnoll, "Lectures on holographic methods for condensed matter physics," arXiv:0903.3246 [hep-th].

C. P. Herzog, "Lectures on Holographic Superfluidity and Superconductivity," J. Phys. A 42, 343001 (2009) [arXiv:0904.1975 [hep-th]].

J. McGreevy, "Holographic duality with a view toward many-body physics," arXiv:0909.0518 [hep-th].

[6] D. T. Son, "Toward an AdS/cold atoms correspondence: a geometric realization of the Schroedinger symmetry," Phys. Rev. D 78, 046003 (2008) arXiv:0804.3972 [hep-th]]. 
[7] K. Balasubramanian and J. McGreevy, "Gravity duals for non-relativistic CFTs," Phys. Rev. Lett. 101, 061601 (2008) arXiv:0804.4053 [hep-th]].

[8] C. P. Herzog, M. Rangamani and S. F. Ross, "Heating up Galilean holography," JHEP 0811, 080 (2008) arXiv:0807.1099 [hep-th]].

[9] J. Maldacena, D. Martelli and Y. Tachikawa, "Comments on string theory backgrounds with non-relativistic conformal symmetry," JHEP 0810, 072 (2008) arXiv:0807.1100 [hep-th]].

[10] A. Adams, K. Balasubramanian and J. McGreevy, "Hot Spacetimes for Cold Atoms," JHEP 0811, 059 (2008) [arXiv:0807.1111 [hep-th]].

[11] S. Kachru, X. Liu and M. Mulligan, "Gravity Duals of Lifshitz-like Fixed Points," Phys. Rev. D 78, 106005 (2008) [arXiv:0808.1725 [hep-th]].

[12] U. H. Danielsson and L. Thorlacius, "Black holes in asymptotically Lifshitz spacetime," JHEP 0903, 070 (2009) arXiv:0812.5088 [hep-th]].

[13] R. B. Mann, "Lifshitz Topological Black Holes," JHEP 0906, 075 (2009) arXiv:0905.1136 [hep-th]].

[14] G. Bertoldi, B. A. Burrington and A. Peet, "Black Holes in asymptotically Lifshitz spacetimes with arbitrary critical exponent," arXiv:0905.3183 [hep-th].

[15] G. Bertoldi, B. A. Burrington and A. W. Peet, "Thermodynamics of black branes in asymptotically Lifshitz spacetimes," arXiv:0907.4755 [hep-th].

[16] M. Taylor, "Non-relativistic holography," arXiv:0812.0530 [hep-th].

[17] D. W. Pang, "A Note on Black Holes in Asymptotically Lifshitz Spacetime," arXiv:0905.2678 [hep-th].

K. Balasubramanian and J. McGreevy, "An analytic Lifshitz black hole," arXiv:0909.0263 [hep-th].

E. Ayon-Beato, A. Garbarz, G. Giribet and M. Hassaine, "Lifshitz Black Hole in Three Dimensions," arXiv:0909.1347 [hep-th].

R. G. Cai, Y. Liu and Y. W. Sun, "A Lifshitz Black Hole in Four Dimensional $R^{2}$ Gravity," JHEP 0910, 080 (2009) [arXiv:0909.2807 [hep-th]]. 
S. J. Sin, S. S. Xu and Y. Zhou, "Holographic Superconductor for a Lifshitz fixed point," arXiv:0909.4857 [hep-th].

Y. S. Myung, Y. W. Kim and Y. J. Park, "Dilaton gravity approach to three dimensional Lifshitz black hole," arXiv:0910.4428 [hep-th].

[18] T. Azeyanagi, W. Li and T. Takayanagi, "On String Theory Duals of Lifshitz-like Fixed Points," JHEP 0906, 084 (2009) [arXiv:0905.0688 [hep-th]].

[19] W. Li, T. Nishioka and T. Takayanagi, "Some No-go Theorems for String Duals of Non-relativistic Lifshitz-like Theories," JHEP 0910, 015 (2009) arXiv:0908.0363 [hep-th]].

[20] E. J. Brynjolfsson, U. H. Danielsson, L. Thorlacius and T. Zingg, "Holographic Superconductors with Lifshitz Scaling," arXiv:0908.2611 [hep-th].

[21] S. W. Hawking and G. T. Horowitz, "The Gravitational Hamiltonian, action, entropy and surface terms," Class. Quant. Grav. 13, 1487 (1996) arXiv:gr-qc/9501014.

[22] S. F. Ross and O. Saremi, "Holographic stress tensor for non-relativistic theories," JHEP 0909, 009 (2009) arXiv:0907.1846 [hep-th]].

[23] Y. Kats and P. Petrov, "Effect of curvature squared corrections in AdS on the viscosity of the dual gauge theory," JHEP 0901, 044 (2009) [arXiv:0712.0743 [hep-th]].

[24] A. Adams, A. Maloney, A. Sinha and S. E. Vazquez, "1/N Effects in Non-Relativistic Gauge-Gravity Duality," JHEP 0903, 097 (2009) [arXiv:0812.0166 [hep-th]].

[25] P. Kovtun, D. T. Son and A. O. Starinets, "Viscosity in strongly interacting quantum field theories from black hole physics," Phys. Rev. Lett. 94, 111601 (2005) arXiv:hep-th/0405231.

[26] M. Brigante, H. Liu, R. C. Myers, S. Shenker and S. Yaida, "Viscosity Bound Violation in Higher Derivative Gravity," Phys. Rev. D 77, 126006 (2008) arXiv:0712.0805 [hep-th]].

[27] M. Brigante, H. Liu, R. C. Myers, S. Shenker and S. Yaida, "The Viscosity Bound and Causality Violation," Phys. Rev. Lett. 100, 191601 (2008) [arXiv:0802.3318 [hep-th]]. 
[28] R. C. Myers, M. F. Paulos and A. Sinha, "Quantum corrections to eta/s," Phys. Rev. D 79, 041901 (2009) [arXiv:0806.2156 [hep-th]].

[29] A. Buchel, R. C. Myers and A. Sinha, "Beyond eta/s = 1/4pi," JHEP 0903, 084 (2009) arXiv:0812.2521 [hep-th]].

[30] I. P. Neupane and N. Dadhich, "Entropy bound and causality violation in higher curvature gravity," Class. Quant. Grav. 26, 015013 (2009).

X. H. Ge, Y. Matsuo, F. W. Shu, S. J. Sin and T. Tsukioka, "Viscosity Bound, Causality Violation and Instability with Stringy Correction and Charge," JHEP 0810, 009 (2008) arXiv:0808.2354 [hep-th]].

N. Banerjee and S. Dutta, "Shear Viscosity to Entropy Density Ratio in Six Derivative Gravity," JHEP 0907, 024 (2009) [arXiv:0903.3925 [hep-th]].

I. P. Neupane, "Black Holes, Entropy Bound and Causality Violation," Int. J. Mod. Phys. A 24, 3584 (2009) [arXiv:0904.4805 [gr-qc]].

S. S. Pal, " $\eta / s$ at finite coupling," arXiv:0910.0101 [hep-th].

[31] R. Brustein, D. Gorbonos and M. Hadad, "Wald's entropy is equal to a quarter of the horizon area in units of the effective gravitational coupling," arXiv:0712.3206 [hep-th].

R. Brustein and A. J. M. Medved, "The ratio of shear viscosity to entropy density in generalized theories of gravity," Phys. Rev. D 79, 021901 (2009) arXiv:0808.3498 [hep-th]].

R. Brustein and A. J. M. Medved, "The shear diffusion coefficient for generalized theories of gravity," Phys. Lett. B 671, 119 (2009) [arXiv:0810.2193 [hep-th]].

[32] N. Iqbal and H. Liu, "Universality of the hydrodynamic limit in AdS/CFT and the membrane paradigm," Phys. Rev. D 79, 025023 (2009) [arXiv:0809.3808 [hep-th]].

[33] R. G. Cai, Z. Y. Nie and Y. W. Sun, "Shear Viscosity from Effective Couplings of Gravitons," Phys. Rev. D 78, 126007 (2008) [arXiv:0811.1665 [hep-th]].

[34] R. G. Cai, Z. Y. Nie, N. Ohta and Y. W. Sun, "Shear Viscosity from Gauss-Bonnet Gravity with a Dilaton Coupling," Phys. Rev. D 79, 066004 (2009) arXiv:0901.1421 [hep-th]]. 
[35] N. Banerjee and S. Dutta, "Higher Derivative Corrections to Shear Viscosity from Graviton's Effective Coupling," JHEP 0903, 116 (2009) [arXiv:0901.3848 [hep-th]].

[36] D. W. Pang, " $R^{2}$ Corrections to Asymptotically Lifshitz Spacetimes," JHEP 0910, 031 (2009) arXiv:0908.1272 [hep-th]].

[37] X. H. Ge and S. J. Sin, "Shear viscosity, instability and the upper bound of the Gauss-Bonnet coupling constant," JHEP 0905, 051 (2009) arXiv:0903.2527 [hepth]].

X. H. Ge, S. J. Sin, S. F. Wu and G. H. Yang, "Shear viscosity and instability from third order Lovelock gravity," arXiv:0905.2675 [hep-th].

[38] S. Caron-Huot, P. Kovtun, G. D. Moore, A. Starinets and L. G. Yaffe, "Photon and dilepton production in supersymmetric Yang-Mills plasma," JHEP 0612, 015 (2006) arXiv:hep-th/0607237.

[39] P. Kovtun and A. Ritz, "Universal conductivity and central charges," Phys. Rev. D 78, 066009 (2008) arXiv:0806.0110 [hep-th]].

[40] R. C. Myers, M. F. Paulos and A. Sinha, "Holographic Hydrodynamics with a Chemical Potential," JHEP 0906, 006 (2009) [arXiv:0903.2834 [hep-th]].

[41] M. Edalati, J. I. Jottar and R. G. Leigh, "Transport Coefficients at Zero Temperature from Extremal Black Holes," arXiv:0910.0645 [hep-th].

[42] M. F. Paulos, "Transport coefficients, membrane couplings and universality at extremality," arXiv:0910.4602 [hep-th].

[43] R. G. Cai, Y. Liu and Y. W. Sun, "Transport Coefficients from Extremal GaussBonnet Black Holes," arXiv:0910.4705 [hep-th].

[44] S. K. Chakrabarti, S. Jain and S. Mukherji, "Viscosity to entropy ratio at extremality," arXiv:0910.5132 [hep-th].

[45] N. Banerjee and S. Dutta, "Near-Horizon Analysis of $\eta / s$, arXiv:0911.0557 [hep-th]. 